\title{
A Review of Techniques for Detection of Movement Intention Using Movement-Related Cortical Potentials
}

\author{
Aqsa Shakeel, ${ }^{1}$ Muhammad Samran Navid, ${ }^{1}$ Muhammad Nabeel Anwar, \\ Suleman Mazhar, ${ }^{2}$ Mads Jochumsen, ${ }^{3}$ and Imran Khan Niazi ${ }^{3,4,5}$ \\ ${ }^{1}$ Human Systems Lab, Department of Biomedical Engineering and Sciences, School of Mechanical and Manufacturing Engineering,
National University of Sciences and Technology (NUST), Islamabad 44000, Pakistan
${ }^{2}$ BiSMiL Lab, Computer Science Department, Information Technology University, Lahore 54000, Pakistan
${ }^{3}$ Center for Sensory-Motor Interaction, Department of Health Science and Technology, Aalborg University, 9100 Aalborg, Denmark
${ }^{4}$ Center for Chiropractic Research, New Zealand College of Chiropractic, 1060 Auckland, New Zealand
${ }_{5}^{5}$ Faculty of Health \& Environmental Sciences, Health \& Rehabilitation Research Institute, Auckland University of Technology,
1010 Auckland, New Zealand
}

Correspondence should be addressed to Muhammad Nabeel Anwar; nabeel@smme.nust.edu.pk

Received 17 September 2015; Revised 23 November 2015; Accepted 2 December 2015

Academic Editor: Anne Humeau-Heurtier

Copyright (C) 2015 Aqsa Shakeel et al. This is an open access article distributed under the Creative Commons Attribution License, which permits unrestricted use, distribution, and reproduction in any medium, provided the original work is properly cited.

\begin{abstract}
The movement-related cortical potential (MRCP) is a low-frequency negative shift in the electroencephalography (EEG) recording that takes place about 2 seconds prior to voluntary movement production. MRCP replicates the cortical processes employed in planning and preparation of movement. In this study, we recapitulate the features such as signal's acquisition, processing, and enhancement and different electrode montages used for EEG data recoding from different studies that used MRCPs to predict the upcoming real or imaginary movement. An authentic identification of human movement intention, accompanying the knowledge of the limb engaged in the performance and its direction of movement, has a potential implication in the control of external devices. This information could be helpful in development of a proficient patient-driven rehabilitation tool based on brain-computer interfaces (BCIs). Such a BCI paradigm with shorter response time appears more natural to the amputees and can also induce plasticity in brain. Along with different training schedules, this can lead to restoration of motor control in stroke patients.
\end{abstract}

\section{Introduction}

The idea of predicting the motor tasks was initially presented by Helmholtz in 1867. Later on, in the fifties Sperry and Von Holst expressed that motor commands make an internal replica which uncovers the anticipated movement and its subsequent sensations [1-3]. From that point forward, the thought of predicting the results of motor tasks by humans has risen as a conspicuous theory in all features of sensorimotor commands.

The brain's current motor activity can be understood in real time through EEG, which can be further employed for prediction of the next voluntary motor task. Real-time EEG might present novel nonmuscular control channel Brain Computer Interfaces (BCIs) for delivering messages and commands to the external world [4]. The immediate objective of BCIs is to provide completely paralyzed users with basic communication capabilities and determine their intent from a range of different electrophysiological signals [4]. Furthermore, research has demonstrated great prospective in the study of brain rhythms and event-related potentials (ERPs) recorded by EEG. Therefore, understanding and analysis of the brain rhythms and ERPs can be used to predict the future motor activity and can be utilized for rehabilitation of physically impaired persons [5].

Studies have shown that EEG comprises enough real-time information to be utilized for different purposes/tasks such as internet browsing, controlling environment (e.g., light, television, and temperature), word processing, controlling a two-dimensional cursor movement on screen, or even 
operating neuroprosthesis [4]. Tasks can be designed which can be used for neurorehabilitation of patients affected with neurodegenerative diseases such as amyotrophic lateral sclerosis [6] and other traumatic brain disorders like stroke [7].

The concept of "premovement" or "before the movement" indicates the time when no muscle movement is evident or is unrelated if it occurs, but the subject is fully familiar with the action he is going to perform in the near future. This is also referred to as planning/preparation of the movements. In this time interval (i.e., $0.5-2 \mathrm{~s}$ prior to the movement onset), the cortex is adapted for implementation of action $[8,9]$.

This paper aims to review the different studies which have used movement-related cortical potentials (MRCPs) to predict the upcoming movements. In the next section, we illustrate the key modifications in the EEG data reported prior to the voluntary movement and how the knowledge of these variations can be used to extract information about the forthcoming movement. In each case, we discuss the main foundations of the study and evaluate the EEG setup and protocols. Finally, in the Conclusion, we recapitulate the key ideas with the hope to bring more consideration to the affluence of premovement and premotor imagery EEG.

\section{Detectable Changes in Brain Rhythm before Onset of Movement}

In this section, we summarize the reported changes in EEG prior to the onset of the actual or imagery movement. All the following phenomena have been delineated both when the movement is imagined and when it is actually executed. One or an amalgamation of these progressions is the fundamental spotlight of the studies acquiring features from premotor imagery or premovement period, discussed in Section 3.

2.1. MRCP and Its Components. The implementation of a motor task in humans measured over the primary motor cortex is preceded by a slow decrease in the EEG amplitude (within at least $500 \mathrm{~ms}$ ) and this potential is known as an MRCP [10], as shown in Figure 1. The MRCP produced in corporation with the planning and execution of a cuebased movement is known as contingent negative variation $(\mathrm{CNV})$ [11], and the one generated in response to selfpaced movement is known as Bereitschaftspotential (BP) $[12,13]$. The MRCP is present in real as well as in imaginary volitional movements [10]. The MRCP comprises three events called readiness potential (RP) or BP, motor potential, and movement-monitoring potential (MMP), which are thought to reflect movement planning/preparation, execution, and control of performance, correspondingly $[14,15]$. The MRCP has been further investigated in normal persons as well as in patients diagnosed with Amyotrophic Lateral Sclerosis, tremor, Parkinson's disease, and stroke, supporting the execution of their motor tasks [14, 16-18]. MRCPs associated with imaginary tasks make them useful for rehabilitation in patients obstructed in movements but still with the ability to wish and imagine a movement $[19,20]$.

2.1.1. Bereitschaftspotential. BP or RP is a negative cortical potential which starts to grow around 1.5 to $1 \mathrm{~s}$ prior to

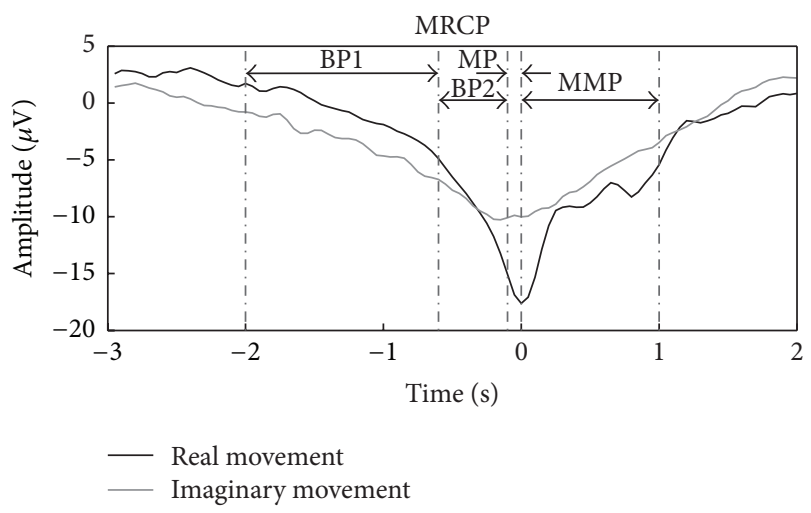

FIGURE 1: MRCPs of a healthy subject for real and imaginary right ankle dorsiflexion. Each wave is an average of 50 large Laplacian spatial filtered EEG trials recorded from sites F3, Fz, F4, C3, $\mathrm{Cz}, \mathrm{C} 4, \mathrm{P} 3, \mathrm{Pz}$, and $\mathrm{P} 4$. Time $0 \mathrm{~s}$ is defined as the movement onset. BP1 is early BP, BP2 is late BP, MP is motor potential, and $\mathrm{MMP}$ is movement-monitoring potential. For more information on experiment protocol, see [23].

the onset of a voluntary movement [12, 13]. It has two fundamental segments: the first part is a slow-rising negative segment which develops about $1.5 \mathrm{~s}$ before the movement onset, known as "early BP," and is more distinguished in the central-medial scalp, while the second part has a steeper slope and happens around 400-500 ms before the movement onset and is called "late BP" which has maximum amplitude over the primary motor cortex [10]. The start of $\mathrm{BP}$ regarding the movement onset varies considerably among different conditions of movement and among subjects [10]. More details on BP can be found in the comprehensive book "The Bereitschaftspotential-Movement Related Cortical Potentials" [21].

2.1.2. Contingent Negative Variation. $\mathrm{CNV}$ is a slow negative wave that originates in the interval (1-1.5 s) between a "Warning" and a "Go" stimulus [11]. It shows expectancy for an imminent signal and preparation for execution of a response. In other words, CNV reveals preparation for signaled movements and is an indicator for anticipation. The earlier part of the $\mathrm{CNV}$ is generated in response to a "Warning" cue and has maximum amplitude over the frontal cortex reflecting phase of the movement, whereas the later or terminal CNV, reflecting preparation for a motor response, begins around $1.5 \mathrm{~s}$ before the "Go" cue and has maximum amplitude over the motor cortex $[3,22]$. The later part of CNV happens even if the subject responds at the time he anticipates the "Go" stimulus [11].

2.2. Generator Sources of MRCP. Several studies reported that the BP might be recorded from subcortical structures such as basal ganglia and thalamus [10]. The work [24] deduced that the early BP was produced by both sensorimotor areas. The work [25] verified that both the ipsilateral and contralateral supplementary motor areas (SMAs) generated potentials consistent with the early BP. 
In order to elucidate the exact area and timing of the motor cortical activation in voluntary movement, dipole source analysis incorporating multiple constraints was applied for MRCP. The work [26] suggested that medial frontocentral (MFC) and sensorimotor areas (SM1) were probable generators of MRCP. The strength of the six dipoles, seeded at the activated spots (three dipoles in left SM1, two in right SM1, and one in MFC) revealed by fMRI, was measured over time. Inside the bilateral SM1, activation of the precentral gyrus happens bilaterally with comparable strength from $-1.2 \mathrm{~s}$, taken after by that of the precentral bank from $-0.5 \mathrm{~s}$ with contralateral dominance through movement execution [26]. Consequently, the postcentral bank gets active just on the contralateral side at $0.1 \mathrm{~s}$ after movement. Activation of the MFC shows timing similar to bilateral precentral gyri. The strength and timing of arousal in the ipsilateral precentral gyrus were like those in the contralateral precentral gyrus and the MFC. But the ipsilateral precentral bank demonstrated much lesser strength than the contralateral precentral bank [26].

To a certain degree, automatic movements such as blinking of eyelids, spontaneous eye movements, swallowing, chewing, and respiration are also controlled by volitional factors; therefore, BP is recorded when these movements are reiterated at a self-paced rate [10]. Self-paced finger movements were related with activation of the anterior SMA, both contralateral sensorimotor cortex and the lateral premotor cortices, but without substantial activation of the ipsilateral sensorimotor cortex [27]. For externally triggered movement, a premovement potential preceding the stimulus was present [27]. Similarly, there were few distinctions in the areas of activation between externally triggered activations and self-paced activation. For a self-paced finger movement, [28] reported SMA activation anteceded that of the motor cortex by $800 \mathrm{~ms}$.

The dorsal premotor cortex (PMd) is believed to play substantial role in cued movement preparation rather than in self-initiated movements [29]. The terminal CNV is generated in the prefrontal cortex including PMd, while the late BP is generated in the primary motor cortex, SMA, and primary somatosensory cortex [29]. This study discovered the effects of variation of PMd on BP and CNV reflecting selfinitiated versus cued movement preparation by increasing and decreasing the excitability of brain using $5 \mathrm{~Hz}$ and $1 \mathrm{~Hz}$ repetitive transcranial magnetic stimulations (rTMS), respectively. They found that rTMS of the left PMd resulted in variation of terminal CNV but not late BP while rTMS of the SMA proper resulted in a modification of late BP but not terminal CNV. This provided evidence that neuronal activity of the left PMd in humans is favorably included in the preparation of externally cued movements as compared to self-initiated movements, contrasting with an opposite role of the SMA proper.

Comparing the MRCP for a foot movement with hand movement showed interesting differences across some movement components [30]. For the hand movement, the late BP is highest over the contralateral central area (approximately $\mathrm{C} 1$ or $\mathrm{C} 2$ of the International 10-20 System) and for the foot movement, late $\mathrm{BP}$ is maximal at the midline (approximately Cz) [30].

2.3. Recording $M R C P$. For the study of $\mathrm{BP}$ in individual subjects against hand movements, it is vital to record EEG from multiple electrodes, including $\mathrm{C} 1$ and $\mathrm{C} 2$, for identifying the abrupt increase of the gradient [30]. The main recording locations for MRCPs are C3, Cz, and C4 [31]. Different studies used different number of electrodes and locations for recording $\mathrm{CNV}$, for example, $\mathrm{C} 3, \mathrm{Cz}$, and $\mathrm{C} 4[32], \mathrm{Fz}, \mathrm{Cz}, \mathrm{Pz}$, $\mathrm{C} 3$, and $\mathrm{C} 4$ [33], and only $\mathrm{Oz}$ [34].

The MRCP can easily be masked by activity in the higher frequency bands because its amplitude typically lies between 5 and $30 \mu \mathrm{V}$ and only occurs at frequencies of around 0 $5 \mathrm{~Hz}$ [31]. Several recordings of the same trials must be taken and then averaged across these trials for meaningful extraction of the MRCP from EEG traces [35]. The reason behind this approach is that EEG data recorded from a single trial contains both the MRCP waveform and spontaneous, random noise [36]. By averaging, the background noise in each trial will be cancelled out, leaving only the MRCP when the data from multiple trials is filtered to eliminate the higher frequency activity and averaged together.

2.4. Factors Influencing BP. Components of MRCP can be inspired by various factors such as preparatory state, level of intention, movement selection, pace of movement repetition, speed and precision of movement, praxis movement, perceived effort, force exerted, discreteness and complexity of movement, learning and skill acquisition, and pathological injuries of various brain structures. The review by [10] sums up several factors influencing BP. Recently, few studies intended to analyze the effect of kinetics of movement such as force and speed on MRCPs [37-40].

\section{Prediction of Intention of Movement}

In this section, we extracted information of the premovement or preimageries from different studies. Some studies display the usefulness of data obtained in the real-time BCIs. Studies utilized different EEG data acquisition techniques including different electrode montages and signal enhancement methods, since these are related to the results reported. Studies mentioned in Tables 1 and 2 try to answer the question whether or not the subject would like to move in the short future. These studies verify that by using MRCPs with the right EEG setting and signal processing techniques, major information can be deduced about the movement yet to come. This section briefly describes some classifiers, filters, and performance metrics used in the studies mentioned in Table 2.

3.1. Classification Algorithms. This section briefly describes the classification algorithms used in studies mentioned in this paper. The classification algorithms include Support Vector Machine (SVM), Linear Discriminant Analysis (LDA), Neural Networks (NN), Multilayer Perceptron (MLP), Bayesian Classifier (BC), $k$ nearest neighbors $(k \mathrm{NN})$, and Mahalanobis Distance (MD). Some of these classification techniques have 
TABLE 1: Experiment protocols of studies reviewed.

\begin{tabular}{|c|c|c|c|c|c|}
\hline Reference & $\begin{array}{l}\text { Number of } \\
\text { subjects }\end{array}$ & $\begin{array}{l}\text { Number of } \\
\text { electrodes }\end{array}$ & Movement type & $\begin{array}{c}\text { Self-paced or } \\
\text { cue-based }\end{array}$ & Brain signals \\
\hline $\begin{array}{l}\text { (Yom-Tov and } \\
\text { Inbar, 2003) [43] }\end{array}$ & 5 (healthy) & $\begin{array}{c}\text { 9, } 4 \text { out of } 9 \\
\text { channels were } \\
\text { used }\end{array}$ & $\begin{array}{c}\text { Executed finger } \\
\text { movement (button } \\
\text { press) }\end{array}$ & Self-paced & MRPs \\
\hline $\begin{array}{l}\text { (Haw et al., 2006) } \\
{[60]}\end{array}$ & $\begin{array}{c}5 \text { (not } \\
\text { mentioned) }\end{array}$ & 1 & $\begin{array}{l}\text { Executed finger } \\
\text { movements }\end{array}$ & Cue-based & $\mathrm{BP}$ \\
\hline $\begin{array}{l}\text { (Bai et al., 2007) } \\
\text { [61] }\end{array}$ & 12 (healthy) & 122 & $\begin{array}{l}\text { Executed hand } \\
\text { movement }\end{array}$ & Self-paced & $\begin{array}{c}\text { MRCPs and ERD } \\
\text { (event-related } \\
\text { desynchronization) }\end{array}$ \\
\hline $\begin{array}{l}\text { (Boye et al., 2008) } \\
\text { [53] }\end{array}$ & $\begin{array}{c}1 \text { (not } \\
\text { mentioned) }\end{array}$ & 9 & $\begin{array}{c}\text { Executed and } \\
\text { imagined foot } \\
\text { movement (isometric } \\
\text { plantar-flexion), but } \\
\text { only imaginary task } \\
\text { was further analyzed }\end{array}$ & Cue-based & MRCP \\
\hline $\begin{array}{l}\text { (Kato et al., 2011) } \\
{[34]}\end{array}$ & $\begin{array}{c}7 \text { (not } \\
\text { mentioned) }\end{array}$ & 1 & $\begin{array}{c}\text { Executed and } \\
\text { imagined finger } \\
\text { movements (button } \\
\text { press) }\end{array}$ & Cue-based & $\mathrm{CNV}$ \\
\hline $\begin{array}{l}\text { (Niazi et al., 2011) } \\
{[42]}\end{array}$ & $\begin{array}{l}19 \text { (healthy) } \\
\text { and } 5 \text { (stroke } \\
\text { patients) }\end{array}$ & 10 & $\begin{array}{c}\text { Executed and } \\
\text { imagined foot } \\
\text { movement (ankle } \\
\text { dorsiflexion) }\end{array}$ & Self-paced & $\mathrm{BP}$ \\
\hline $\begin{array}{l}\text { (Lew et al., 2012) } \\
{[63]}\end{array}$ & $\begin{array}{l}8 \text { (healthy), } 2 \\
\text { (control), and } \\
2 \text { (stroke } \\
\text { patients) } \\
\end{array}$ & $\begin{array}{c}\text { 64, } 34 \text { out of } 64 \\
\text { channels were } \\
\text { used }\end{array}$ & $\begin{array}{c}\text { Executed arm } \\
\text { movements (reaching } \\
\text { task) }\end{array}$ & Self-paced & $\mathrm{BP}$ \\
\hline $\begin{array}{l}\text { (Niazi et al., 2012) } \\
{[19]}\end{array}$ & 16 (healthy) & 10 & $\begin{array}{l}\text { Imagined foot } \\
\text { movements } \\
\text { (dorsiflexion) } \\
\end{array}$ & Self-paced & MRCP \\
\hline $\begin{array}{l}\text { (Niazi et al., 2013) } \\
{[65]}\end{array}$ & $\begin{array}{l}20 \text { (healthy) } \\
\text { and } 5 \text { (stroke } \\
\text { patients) }\end{array}$ & 10 & $\begin{array}{l}\text { Executed and } \\
\text { imagined foot } \\
\text { movements } \\
\text { (dorsiflexion) }\end{array}$ & Self-paced & MRCP \\
\hline $\begin{array}{l}\text { (Ahmadian et al., } \\
\text { 2013) [64] }\end{array}$ & 3 (healthy) & 128 channels & $\begin{array}{c}\text { Finger movement } \\
\text { (button press) }\end{array}$ & Self-paced & $\mathrm{BP}$ \\
\hline $\begin{array}{l}\text { (Jochumsen et al., } \\
\text { 2013) [39] }\end{array}$ & 12 (healthy) & 10 & $\begin{array}{c}\text { Executed foot } \\
\text { movement (isometric } \\
\text { dorsiflexion) }\end{array}$ & Cue-based & MRCP \\
\hline $\begin{array}{l}\text { (Jiang et al., 2015) } \\
\text { [66] }\end{array}$ & 9 (healthy) & 9 & $\begin{array}{l}\text { Executed foot } \\
\text { movements } \\
\text { (stepping) }\end{array}$ & Self-paced & MRCP \\
\hline $\begin{array}{l}\text { (Xu et al., 2014) } \\
{[20]}\end{array}$ & 9 (healthy) & 9 & $\begin{array}{c}\text { Executed and imagery } \\
\text { foot movements } \\
\text { (dorsiflexion) }\end{array}$ & Self-paced & MRCP \\
\hline
\end{tabular}

been reviewed in detail by [41]. The details of Matched Filter technique can be found in [42] and for Locality Preserving Projection (LPP) in [20].

3.1.1. Support Vector Machine. The Support Vector Machine (SVM) is a pattern recognition algorithm that has been successfully applied to wide variety of classification problems. It learns to distinguish among various classes of objects by some complex data transformations and then separate the data based on the defined labels for classes. For example, the data for a two-class problem consist of objects labeled corresponding to two classes, for example, +1 (data belong to class 1 ) or -1 (data belong to class two). The system then automatically identifies the input points and uses them to represent the solution [43].

3.1.2. Linear Discriminant Analysis. The purpose of Linear Discriminant Analysis (LDA) (also called Fisher's LDA) is to use hyperplanes to isolate the data into different classes [44]. The segregating hyperplane is acquired by seeking the 


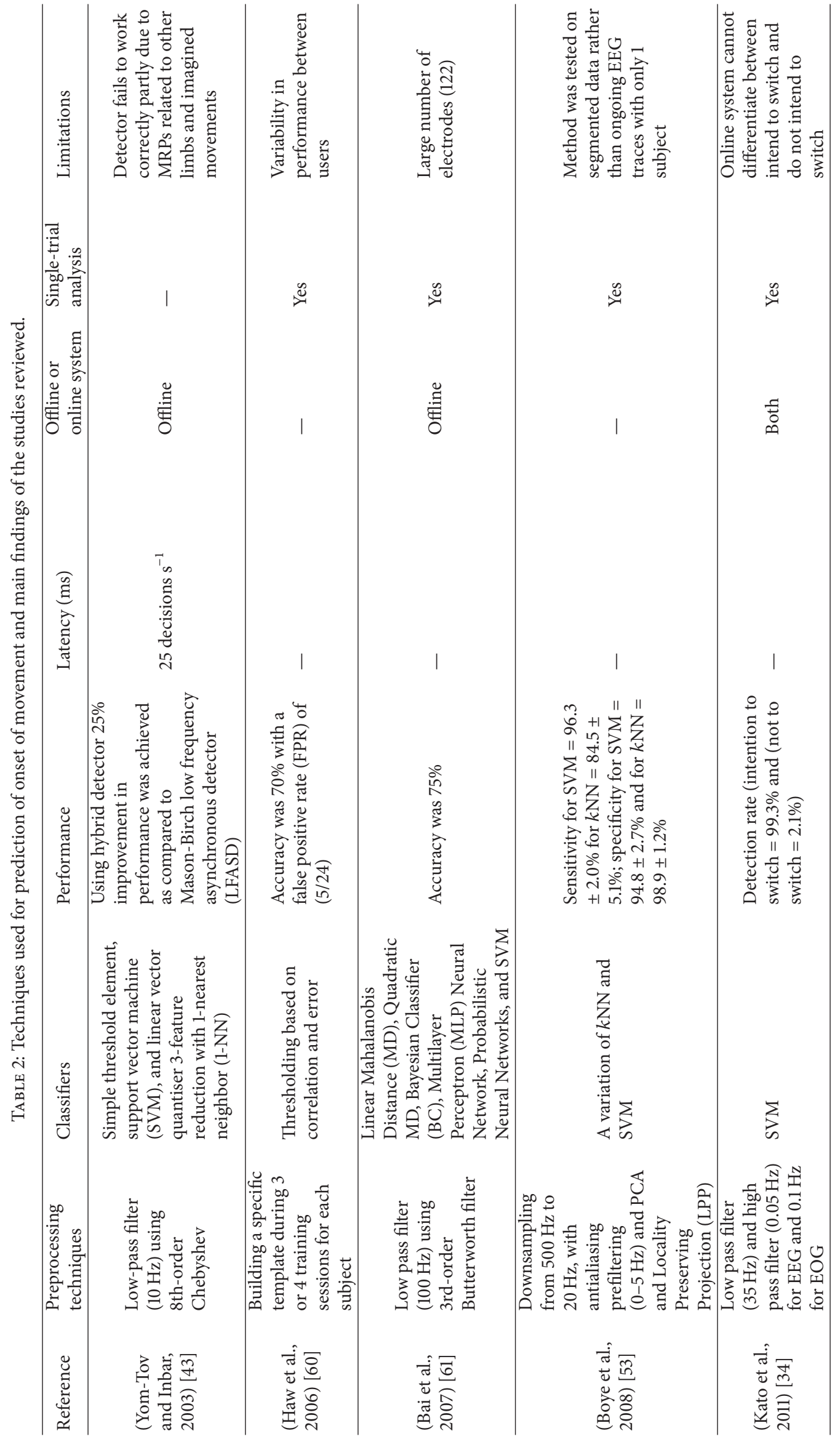




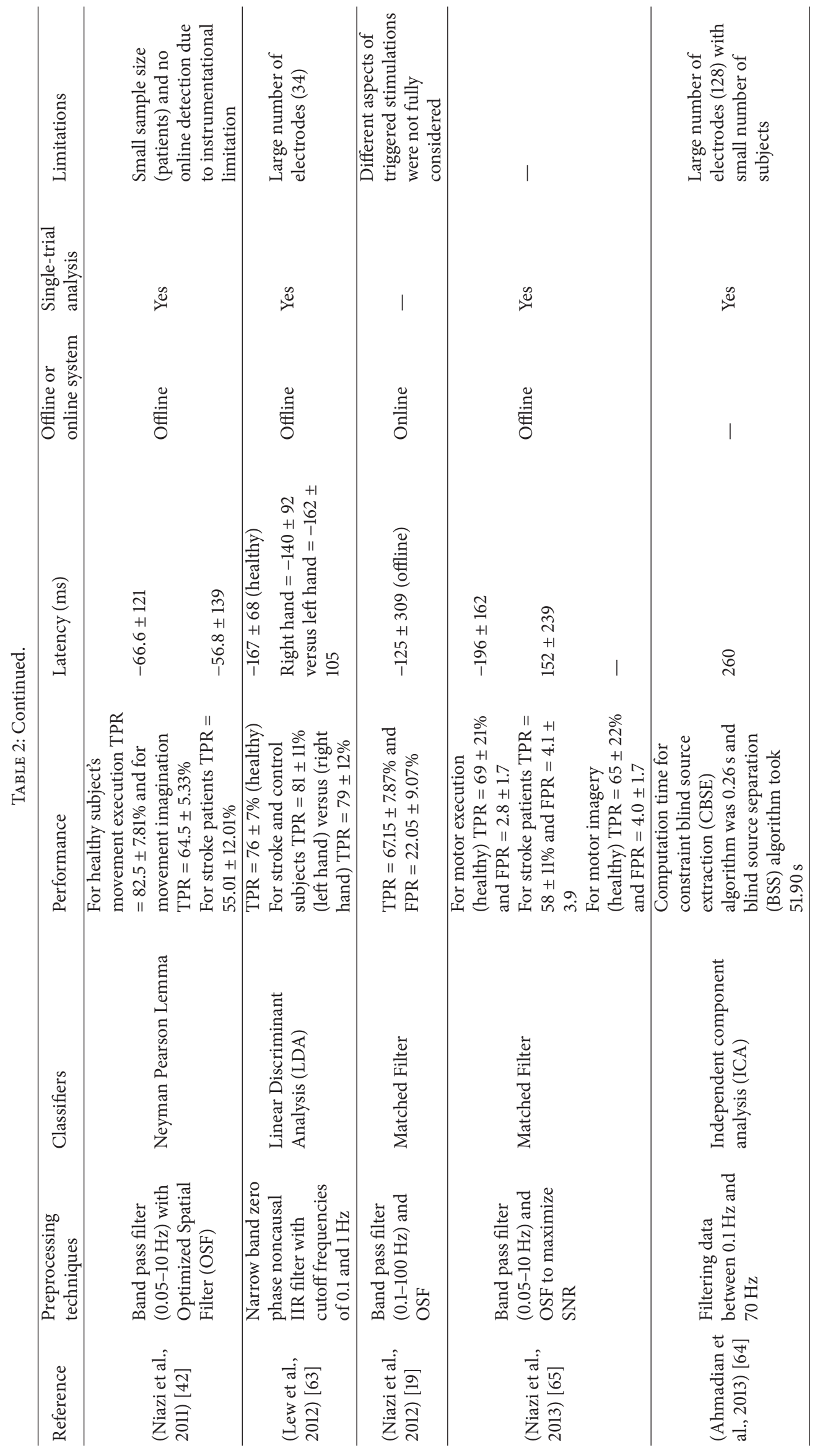




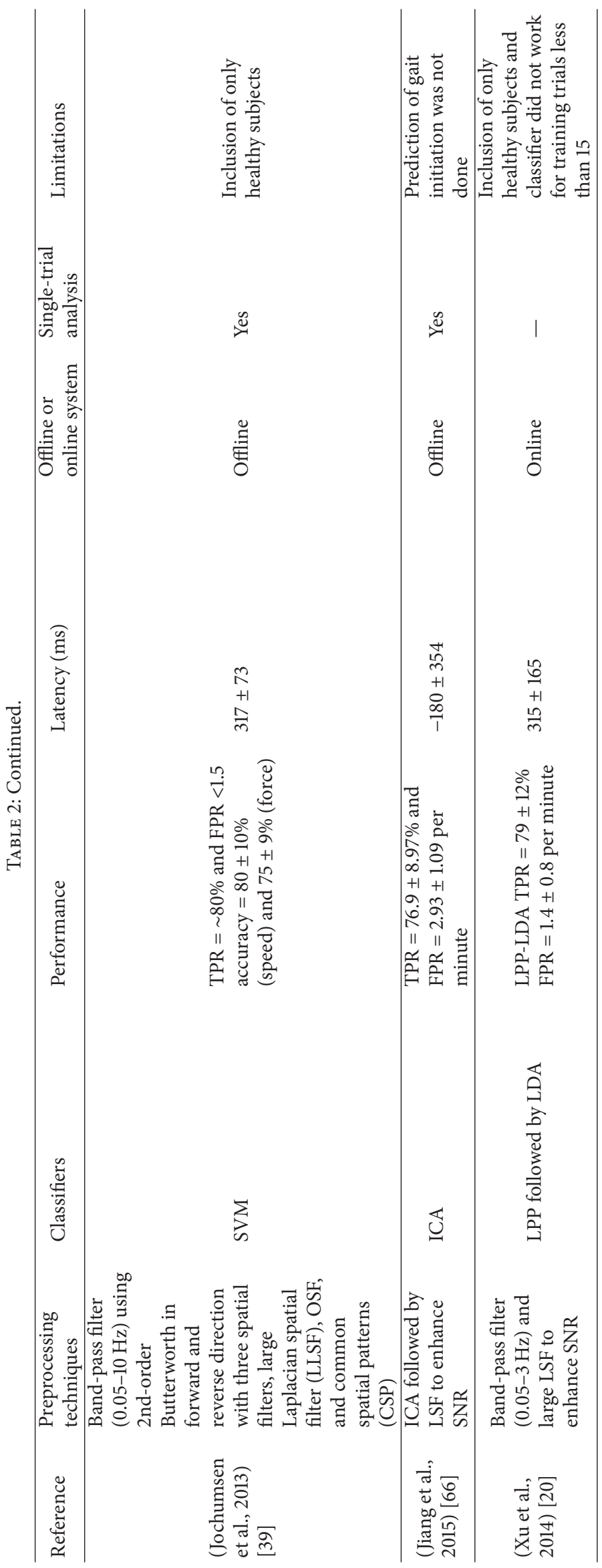


projection that decreases the interclass variance and increases the distance between the two classes' means. To solve a twoclass problem the isolation of the input data vector into either class depends on presence of the data vector on which side of the hyperplane [44].

3.1.3. Neural Networks. Neural Networks (NN) can be thought as circuits of immensely interconnected units with flexible interconnection weights, which allow us to yield nonlinear decision boundaries. They can be classified by architecture, algorithm for calibrating the weights, and the kind of units utilized as a part of the circuit [45]. Most widely used NN is the MLP.

3.1.4. Multilayer Perceptron. Multilayer Perceptron (MLP) is composed of several layers of neurons: an input layer, perhaps one or many hidden layers, and an output layer [45]. Each input layer is connected with the output of the previous layer, while the neurons of the output layer deduce the class of the input feature vector. MLP can approximate any continuous function when it is composed of enough neurons and layers. It can also classify any number of classes, which makes MLP very flexible classifiers and adaptive to a variety of problems [46].

3.1.5. Bayesian Classifiers. Bayesian Classifier (BC) depends on Bayes' theorem and can anticipate class membership probabilities, for example, the likelihood that a given sample fits into a specific class. In order to classify a feature vector, it learns the way of computing the probability of each class. $\mathrm{BC}$ assumes that estimation of a specific feature does not rely on value of any other feature, which provided the class variable. Being a generative classifier, it produces nonlinear decision boundaries and performs more efficient rejection of uncertain samples as compared to discriminative classifiers [44].

3.1.6. $k$ Nearest Neighbors. The objective of this method is to allocate to an unseen point the dominant class amongst its $k$ nearest neighbors within the training set. $k \mathrm{NN}$ can approximate any function with enough training samples and a sufficiently high value of $k$, which allows it to yield nonlinear decision boundaries [47].

3.1.7. Mahalanobis Distance. Mahalanobis Distance assigns a feature vector to a class according to its nearest neighbor(s) from a class prototype. It assumes a Gaussian distribution $N(\mu c, M c)$ for each prototype of the class $c$. Then a feature vector $x$ is allocated to the class that links to the nearest prototype [48]:

$$
d_{c}(x)=\sqrt{\left(x-\mu_{c}\right) M_{c}^{-1}\left(x-\mu_{c}\right)^{T}} .
$$

3.2. Spatial Filters. A spatial filter amalgamates data from two or more locations (electrodes). Spatial filtering techniques comprise common spatial patterns (CSP), common average referencing (CAR), surface Laplacian (SL), independent component analysis (ICA), and principle component analysis
(PCA). This section briefly describes some spatial filters; for more details please refer to [49].

ICA is a method intended to find a linear illustration of non-Gaussian data in the form of statistically independent constituent components [50]. Measured signals comprising a linear mixture of statistically independent source signals can be dissolved into their vital Independent Components (ICs), hence deducing the original source signals using ICA [50, 51]. ICA finds the weighting of the channels from the data like PCA and CSP [4], while CAR and SL amalgamate channels linearly to produce a set of weights that does not depend on the underlying data [4]. SL highlights the radial component of the neural activity placed directly below each recording electrode from sources, whereas ICA is capable of detecting both radial and tangential sources and consequently may be beneficial over SL $[4,52]$. The details regarding CSP and CAR can be found in [49].

3.3. Performance Measures. The performance of studies is computed using sensitivity, specificity, and detection error. Sensitivity (also known as true positive rate (TPR)) quantifies the fraction of actual positives (movements) which are precisely recognized. Specificity (also called the true negative rate (TNR)) assesses the fraction of negatives (no motion or noise) which are exactly detected. Sensitivity and specificity are calculated using the following equations, respectively, where TP and TN represent number of true positives and number of true negatives, respectively [53]:

$$
\begin{aligned}
& \text { Sensitivity }=\frac{\mathrm{TP}}{\mathrm{TP}+\mathrm{FN}}, \\
& \text { Specificity }=\frac{\mathrm{TN}}{\mathrm{TN}+\mathrm{FP}} .
\end{aligned}
$$

\section{Studies for Predicting the Intention of Movement}

For the development of self-paced closed loop BCIs, the robust detection of motor intention is a vital and critical issue. In the past decades, sensory motor rhythms have been used for detection of motor intention in studies comprising BCIs to control visual feedback [54] or trigger external devices [55] without investigating latency. In the initial studies, the acceptable delay in control has not been considered in detail for BCI control applications. In other fields, for example, multifunction prostheses control by myoelectric signals, a $200 \mathrm{~ms}$ delay is considered acceptable [56-58]. To induce plasticity in BCI-based neurorehabilitation applications, it was demonstrated that the required delay was in the same range as for control, that is, in the order of a few hundred milliseconds [59]. Therefore, a reliable detection with minimal latency and high accuracy would play a vital role in an effective BCI rehabilitation tool [7].

4.1. Techniques Utilized and Performance. In recent years, slow cortical potentials captured the attention in the rehabilitation field. Several studies have been reported, which 
concentrated their attention intended for communication purposes.

Yom-Tov and Inbar [43] developed an algorithm combining Matched Filter, a nonlinear transformation, and a classifier to detect MRCP using small number of EEG channels. The algorithm was compared with Mason-Birch lowfrequency asynchronous detector (LFASD) and optimum detector by both offline evaluation and theoretical analysis. The algorithm used by this study showed $25 \%$ improvement and the detector worked at a rate of 25 decisions $\mathrm{s}^{-1}$ as compared to 16 decisions s ${ }^{-1}$ in the LFASD. But the detector may not be useful in every application and failed to operate correctly, partly due to interference of MRPs from other limbs of the body and possibly due to imagined movements. These verdicts indicate that correctly identified features have a major role not only in discarding MRCPs due to movements that are not part of the BCI system, but also in determining the limb moved (or imagined movement) by the user. During sessions, training the subject to reduce body movement can probably attain better results.

To detect movement planning, [60] employed a userspecific template matching structure as part of a method. In this study, the emphasis was more on movement detection than the prediction. Performing actual finger movement with cues, one electrode recorded BP waveform which was then used to build the template. However, inconsistency in performance between subjects was evident in this study which needs more investigation or perhaps a different methodology.

From single trial EEG, [61] showed that effectual combinations of computational methods can deliver possible classification of human movement intention using large number of electrodes. The combinations of temporal filtering using power spectral density estimation and discrete wavelet transform, spatial filtering using ICA and surface Laplacian derivation, and classification methods using LMD, QMD, BC, and SVM provided higher performance than those of other combinations. Evaluation is recommended in order to check whether performance can be enhanced after training with feedback or not.

The validity of OSF on imagination of isometric plantarflexion was confirmed in a study conducted by [53]. Features were extracted with PCA and classification was performed using $k \mathrm{NN}$ and SVM. In this study, the TPRs were high (80$90 \%$ ) but the method was verified on segmented data instead of ongoing EEG traces with one subject only.

Kato et al. [34] designed a BCI master switch by detecting the CNV related potentials and performed both offline and online studies. In order to ameliorate the single-trial discrimination of user intentions to switch, CNV was employed due to its high SNR. Using only one electrode and performing four cued button press tasks, they also applied SVM to improve the single-trial detection of CNV-related potentials. Their online system did not discern between "intend to switch" and "do not intend to switch." This was maybe because of using default parameters of SVM in LIBSVM for the distinction of CNV-related potentials $[34,62]$.

The detection of movement intention from single trial MRCPs of movement imagination and movement execution was performed by [42]. The task performed by the subject was always the same (ankle dorsiflexion). They performed offline detection due to instrumentation limitation and provided the feasibility of the approach in stroke patients, along with the extensive analysis in healthy subjects. The accuracy of the detection of movement intention was measured by applying a similar spatial filtering technique. In this study, a portion of the negative phase $(2 \mathrm{~s}$ ) of the MRCP was used as a template. In order to improve SNR of MRCP, OSF was used (TPR of $82.5 \pm 7.81 \%)$. OSF outperformed large Laplacian spatial filter (TPR of $68.7 \pm 14.9 \%)$ and CSP $(\mathrm{TPR}=55.4 \pm 14.01 \%)$.

Lew et al. [63] explained that it is possible to predict the movement $500 \mathrm{~ms}$ before its occurrence. For the training phase, the signal prior to movement onset by $500 \mathrm{~ms}$ was used in comparison with $500 \mathrm{~ms}$ before the auditory cue. While a shifting window was implemented for the test phase and LDA was employed, their results showed maximum average TPR of $81 \%$ for left hand while $79 \%$ for right of stroke and control subjects. While for healthy subjects average TPR was $76 \pm 7 \%$ with latency of $-167 \pm 68 \mathrm{~ms}$. This offline study employed large number of electrodes with small sample size of patients.

Ahmadian et al. [64] showed the superiority of CBSE based algorithm in detection of brain potential compared with BSS based algorithm using LDA. Subjects performed cued button preprocessing. CBSE based algorithm took $0.26 \mathrm{~s}$ while BSS based algorithm took 51.90 s. All 128 channels EEG data was employed in the analysis. It was suggested that false detection rate can be reduced if BSS-based algorithm uses extracted sources which are mistaken with the shape of BP from other regions of brain. On the other hand, this amendment would increase the computation time.

Motor intention could be detected from MRCP using the Matched Filter, with small latency and satisfactory accuracy. The same task was performed in $[19,20,42,65]$. Niazi et al. performed analysis on both healthy and stroke patients [42, 65]. He investigated the possibility of eluding the individual training phase in the detection of movement intention. The detection accuracy with the average template for the motor imagery data was $65 \pm 22 \%$ [65] and with the individual template was $60 \pm 13 \%$ [65].

Jochumsen et al. [39] detected movement intentions and extracted distinct levels of speed and force of the intended movements. The temporal features were classified with an optimized SVM. This study evaluated performance when detection was combined with classification. The system correctly detected $81 \%$ of the movements. At the point of detection, system classified $75 \pm 9 \%$ and $80 \pm 10 \%$ when altering the force and speed, respectively. After combination of detector and classifier, the system detected and correctly classified $64 \pm 13 \%$ and $67 \pm 13 \%$ of movements. Incorrectly detected and classified movements were about $21 \pm 7 \%$ and $16 \pm 9 \%$ while latency was $317 \pm 73 \mathrm{~ms}$ before the movement onset. The authors included only healthy subjects and the signals were processed offline. The performance of system will decline if user is a patient due to severity of motor impairment, mood, and amount of training.

$\mathrm{Xu}$ et al. [20] performed analysis on healthy subjects only. In this study, LPP-LDA showed higher accuracy and shorter latency than Matched Filter, having limitation that the 
classifier would not work when training trials were less than 15. The proposed algorithm had similar FPR for imagination and execution across all subjects. TPR for execution and imagery was greater than $80 \%$ and $70 \%$ in this study, making it significantly better than those with Matched Filter approach $[19,42,65]$. Also detection latency (315 $\pm 165 \mathrm{~ms})$ was significantly shorter than that with Matched Filter (460 \pm $123 \mathrm{~ms})[19,42,65]$.

To further improve the results, [66] applied ICA and the LSF to improve the signal-to-noise ratio of MRCP. Following these preprocessing steps, Matched Filter was applied to perform single-trial detection of gait initiation. TPR was $76.9 \pm 8.97 \%$, and the false positive rate FPR was $2.93 \pm 1.09$ per minute. On a single trial basis, these results demonstrated the possibility of detecting the intention of gait initiation from EEG signals.

In a recent study by [67], six healthy persons and eight stroke patients performed upper limb self-paced reaching movements. This study used a classifier that combined the information acquired from analysis of the BP and ERD cortical processes. System validation was performed with the combined classifier (ERD and BP patterns) and equivalent classifiers (using either BP or ERD). The results obtained for healthy subjects were similar to [20]. However, the average latencies (healthy: $-89.9 \pm 349.2 \mathrm{~ms}$ and patients: $35.9 \pm 352.3 \mathrm{~ms})$ were less than [20] $(315 \pm 165 \mathrm{~ms})$. These dissimilarities might be due to differences in the way subjects executed the task in each experiment such as changes between upper limb and lower limb cortical patterns, speed of movements among others, and length of the resting intervals between movements. The observed alterations could also be because of the combined use of the ERD and BP features anticipated here, which facilitates reduction in FPR and, as a result, enables the preference of more anticipative detection thresholds [67].

A complexity confronted in this paper involves the absence of similar studies in terms of purpose of detector, signal acquisition, limb movement, and number of electrodes. It should be noted that movements executed in different studies were not similar leading to variances in signal morphology and SNR. Ideally studies should be compared within the same context, that is, with similar protocol, users, and similar extraction of features.

Although the mentioned studies have delivered a valuable insight into the prediction of MRCPs using different signal acquisition techniques, the framework of research is not without its impediments. One limitation relevant to most of the studies mentioned is the absence of clear ecological validity in the research, that is, "the extent to which an experimental situation mimics a real world situation" [31]. Presence of an ecological validity in a study means that the expertise used in the laboratory situation could be as equivalent as possible to the real skill the research is exploring and preferably identical.

4.2. Guidelines to Choose a Classifier. EEG signals are notably nonstationary so training sets acquired from different sessions are probable to be quite different. Consequently, a low variance (sensitivity to training set) can be a solution to tackle with the variability issue in some studies. Unstable classifiers tend to have a low bias (deviation between the estimated mapping and the superlative mapping) and a high variance, while stable classifiers have a high bias and a low variance [41]. This might be an explanation of why some simple classifiers like SVM, Matched Filter, and so forth sometimes surpass more than complex ones. Simple classifiers are generally slower than other classifiers but fast enough for real-time applications. Here the question is whether it is worthy to get higher performance at the expense of computational cost. In order to attain minimal classification error, both the variance and the bias must be small. Unfortunately, natural variancebias tradeoff is always present [41].

The classifier will probably give bad performance if the number of training data is lesser matched to the size of the feature vectors. Usage of at least five to ten times training samples per class as the dimensionality is recommended [68, 69]. Generally the training set is small and dimensionality is high so; unluckily this cannot be useful in all BCI systems. One of the reasons might be the long duration of the tasks, which on the other hand is hectic for subjects. Consequently this "curse" is a key concern in BCI design.

Furthermore, combinations of classifiers also seem to be very efficient in some studies $[20,70,71]$. Normally experiments are performed in a controlled manner minimizing noise and other artifacts while presence of noise in real life scenario is quite obvious. One possible solution might be to increase the generalization abilities of the classifier.

\section{Future Work}

MRCP has been employed as a control signal in BCI technology. It is mainly beneficial for neuromodulation applications in which the delay between the intention of action and the feedback from the system is crucial to induce plasticity [19]. BCIs have primarily been used for control and communication purposes [4]; however, in recent years its prospective in neurorehabilitation has been studied such as functional electrical stimulation [6]. BCIs are extensively used in research and major concern is its long-term effects or long-term changeability of EEG signals to evaluate retention of the plasticity over time [7]. So there is a need to design studies over longer duration to evaluate the performance and accuracy of the BCI system for healthy subjects and patients. As signal processing in BCIs continues to progress, the next perspective is to integrate additional information regarding neurophysiology, disease behaviors and its advancement, and signal dynamics into the existing or future approaches.

\section{Conclusion}

EEG data collected prior to imminent movement which associates with motor preparation and planning period of the brain present substantial prediction potentials. Illustrating the intention to move through MRCP can be employed in rehabilitation protocols. Depending on the purpose of the BCI system, a higher TPR could be achieved increasing the number of false positives, while some studies tend to give a priority to a low FPR rather than high TPRs [43]. In summary, 
this paper reviews the proficiency of EEG in predicting the next motor task and primarily targeted at providing the examples of the progress in this field.

\section{Conflict of Interests}

The authors declare that there is no conflict of interests regarding the publication of this paper.

\section{References}

[1] S. J. Blakemore, S. J. Goodbody, and D. M. Wolpert, "Predicting the consequences of our own actions: the role of sensorimotor context estimation," The Journal of Neuroscience, vol. 18, no. 18, pp. 7511-7518, 1998.

[2] D. M. Wolpert and J. R. Flanagan, "Motor prediction," Current Biology, vol. 11, no. 18, pp. R729-R732, 2001.

[3] P. Ahmadian, S. Cagnoni, and L. Ascari, "How capable is noninvasive EEG data of predicting the next movement? A mini review," Frontiers in Human Neuroscience, vol. 7, article 124, 2013.

[4] J. R. Wolpaw, N. Birbaumer, D. J. McFarland, G. Pfurtscheller, and T. M. Vaughan, "Brain-computer interfaces for communication and control," Clinical Neurophysiology, vol. 113, no. 6, pp. 767-791, 2002.

[5] K. Dremstrup, I. K. Niazi, M. Jochumsen, N. Jiang, N. Mrachacz-Kersting, and D. Farina, "Rehabilitation using a brain computer interface based on movement related cortical potentials-a review," in XIII Mediterranean Conference on Medical and Biological Engineering and Computing 2013, L. M. Roa Romero, Ed., vol. 41 of IFMBE Proceedings, pp. 1659-1662, Springer, Basel, Switzerland, 2014.

[6] J. J. Daly and J. R. Wolpaw, "Brain-computer interfaces in neurological rehabilitation," The Lancet Neurology, vol. 7, no. 11, pp. 1032-1043, 2008.

[7] R. Xu, N. Jiang, N. Mrachacz-Kersting et al., "A closed-loop brain-computer interface triggering an active ankle-foot orthosis for inducing cortical neural plasticity," IEEE Transactions on Biomedical Engineering, vol. 61, no. 7, pp. 2092-2101, 2014.

[8] D. J. Crammond and J. F. Kalaska, "Prior information in motor and premotor cortex: activity during the delay period and effect on pre-movement activity," Journal of Neurophysiology, vol. 84, no. 2, pp. 986-1005, 2000

[9] I. Toni and R. E. Passingham, "Movement preparation: neuroimaging studies," in The Bereitschaftspotential, pp. 269-281, Springer, New York, NY, USA, 2003.

[10] H. Shibasaki and M. Hallett, "What is the Bereitschaftspotential?” Clinical Neurophysiology, vol. 117, no. 11, pp. 2341-2356, 2006.

[11] W. G. Walter, R. Cooper, V. J. Aldridge, W. C. McCallum, and A. L. Winter, "Contingent negative variation : an electric sign of sensori-motor association and expectancy in the human brain," Nature, vol. 203, no. 4943, pp. 380-384, 1964.

[12] H. H. Kornhuber and L. Deecke, "Hirnpotentialänderungen bei willkürbewegungen und passiven bewegungen des menschen: bereitschaftspotential und reafferente potentiale," Pflüger's Archiv für die gesamte Physiologie des Menschen und der Tiere, vol. 284, no. 1, pp. 1-17, 1965.

[13] H. H. Kornhuber and L. Deecke, "Hirnpotentialänderungen bei Willkürbewegungen und passiven Bewegungen des Menschen: Bereitschaftspotential und reafferente Potentiale," Pflügers
Archiv für die Gesamte Physiologie des Menschen und der Tiere, vol. 284, no. 1, pp. 1-17, 1965.

[14] K. Dremstrup, Y. Gu, O. F. D. Nascimento, and D. Farina, "Movement-related cortical potentials and their application in brain-computer interfacing," in Introduction to Neural Engineering for Motor Rehabilitation, pp. 253-266, Wiley, 2013.

[15] O. F. Do Nascimento, K. D. Nielsen, and M. Voigt, "Movementrelated parameters modulate cortical activity during imaginary isometric plantar-flexions," Experimental Brain Research, vol. 171, no. 1, pp. 78-90, 2006.

[16] Y. Gu, D. Farina, A. R. Murguialday, K. Dremstrup, P. Montoya, and N. Birbaumer, "Offline identification of imagined speed of wrist movements in paralyzed ALS patients from single-trial EEG," Frontiers in Neuroscience, vol. 3, 2009.

[17] Y. Gu, D. Farina, A. R. Murguialday, K. Dremstrup, and N. Birbaumer, "Comparison of movement related cortical potential in healthy people and amyotrophic lateral sclerosis patients," Frontiers in Neuroscience, vol. 7, article 65, 2013.

[18] J. Ibáñez, J. I. Serrano, M. D. Del Castillo, and L. J. Barrios, "An asynchronous BMI system for online single-trial detection of movement intention," in Proceedings of the 32nd Annual International Conference of the IEEE Engineering in Medicine and Biology Society (EMBC"10), pp. 4562-4565, IEEE, Buenos Aires, Argentina, September 2010.

[19] I. K. Niazi, N. Mrachacz-Kersting, N. Jiang, K. Dremstrup, and D. Farina, "Peripheral electrical stimulation triggered by selfpaced detection of motor intention enhances motor evoked potentials," IEEE Transactions on Neural Systems and Rehabilitation Engineering, vol. 20, no. 4, pp. 595-604, 2012.

[20] R. Xu, N. Jiang, C. Lin, N. Mrachacz-Kersting, K. Dremstrup, and D. Farina, "Enhanced low-latency detection of motor intention from EEG for closed-loop brain-computer interface applications," IEEE Transactions on Biomedical Engineering, vol. 61, no. 2, pp. 288-296, 2014.

[21] M. Jahanshahi and M. Hallett, The Bereitschaftspotential: Movement-Related Cortical Potentials, Springer, 2003.

[22] C. H. M. Brunia, "CNV and SPN: indices of anticipatory behavior," in The Bereitschaftspotential, pp. 207-227, Springer, New York, NY, USA, 2003.

[23] M. Jochumsen, M. S. Navid, R. W. Nedergaard, M. N. Anwar, I. K. Niazi, and K. Dremstrup, "Online detection and classification of movement kinetics," in Proceedings of the 6th International Brain-Computer Interface Conference, Graz, Austria, September 2014.

[24] R. Neshige, H. Lüders, and H. Shibasaki, "Recording of movement-related potentials from scalp and cortex in man," Brain, vol. 111, no. 3, pp. 719-736, 1988.

[25] A. Ikeda, H. O. Lüders, R. C. Burgess, and H. Shibasaki, "Movement-related potentials recorded from supplementary motor area and primary motor area: role of supplementary motor area in voluntary movements," Brain, vol. 115, no. 4, pp. 1017-1043, 1992.

[26] K. Toma, T. Matsuoka, I. Immisch et al., "Generators of movement-related cortical potentials: fMRI-constrained EEG dipole source analysis," NeuroImage, vol. 17, no. 1, pp. 161-173, 2002.

[27] M. Jahanshahi, I. H. Jenkins, R. G. Brown, C. D. Marsden, R. E. Passingham, and D. J. Brooks, "Self-initiated versus externally triggered movements I. An investigation using measurement of regional cerebral blood flow with PET and movement-related potentials in normal and Parkinson's disease subjects," Brain, vol. 118, no. 4, pp. 913-933, 1995. 
[28] D. Wildgruber, M. Erb, U. Klose, and W. Grodd, "Sequential activation of supplementary motor area and primary motor cortex during self-paced finger movement in human evaluated by functional MRI," Neuroscience Letters, vol. 227, no. 3, pp. 161164, 1997.

[29] M.-K. Lu, N. Arai, C.-H. Tsai, and U. Ziemann, "Movement related cortical potentials of cued versus self-initiated movements: double dissociated modulation by dorsal premotor cortex versus supplementary motor area rTMS," Human Brain Mapping, vol. 33, no. 4, pp. 824-839, 2012.

[30] H. Shibasaki, G. Barrett, E. Halliday, and A. M. Halliday, "Components of the movement-related cortical potential and their scalp topography," Electroencephalography and Clinical Neurophysiology, vol. 49, no. 3-4, pp. 213-226, 1980.

[31] D. J. Wright, P. S. Holmes, and D. Smith, "Using the movementrelated cortical potential to study motor skill learning," Journal of Motor Behavior, vol. 43, no. 3, pp. 193-201, 2011.

[32] S. K. Jankelowitz and J. G. Colebatch, "Movement-related potentials associated with self-paced, cued and imagined arm movements," Experimental Brain Research, vol. 147, no. 1, pp. 98$107,2002$.

[33] T. Nonaka, M. Yoshida, T. Yamaguchi et al., "Contingent negative variations associated with command swallowing in humans," Clinical Neurophysiology, vol. 120, no. 10, pp. 18451851, 2009.

[34] Y. X. Kato, T. Yonemura, K. Samejima, T. Maeda, and H. Ando, "Development of a BCI master switch based on single-trial detection of contingent negative variation related potentials," in Proceedings of the 33rd Annual International Conference of the IEEE Engineering in Medicine and Biology Society (EMBS '11), pp. 4629-4632, IEEE, Boston, Mass, USA, September 2011.

[35] N. Birbaumer, T. Elbert, A. G. M. Canavan, and B. Rockstroh, "Slow potentials of the cerebral cortex and behavior," Physiological Reviews, vol. 70, no. 1, pp. 1-41, 1990.

[36] S. J. Luck, An Introduction to The Event-Related Potential Technique, MIT Press, 2014.

[37] D. Farina, O. F. do Nascimento, M.-F. Lucas, and C. Doncarli, "Optimization of wavelets for classification of movementrelated cortical potentials generated by variation of force-related parameters," Journal of Neuroscience Methods, vol. 162, no. 1-2, pp. 357-363, 2007.

[38] Y. Gu, O. F. Do Nascimento, M.-F. Lucas, and D. Farina, "Identification of task parameters from movement-related cortical potentials," Medical \& Biological Engineering \& Computing, vol. 47, no. 12, pp. 1257-1264, 2009.

[39] M. Jochumsen, I. K. Niazi, N. Mrachacz-Kersting, D. Farina, and K. Dremstrup, "Detection and classification of movementrelated cortical potentials associated with task force and speed," Journal of Neural Engineering, vol. 10, no. 5, Article ID 056015, 2013.

[40] Y. Fu, B. Xu, L. Pei, and H. Li, "Time domain features for relationship between speed and slow potentials activity during periodic movement and motor imagery at fast and slow for BCRI," Procedia Environmental Sciences, vol. 8, pp. 498-505, 2011.

[41] F. Lotte, M. Congedo, A. Lécuyer, F. Lamarche, and B. Arnaldi, "A review of classification algorithms for EEG-based braincomputer interfaces," Journal of Neural Engineering, vol. 4, no. 2, 2007.
[42] I. K. Niazi, N. Jiang, O. Tiberghien, J. F. Nielsen, K. Dremstrup, and D. Farina, "Detection of movement intention from singletrial movement-related cortical potentials," Journal of Neural Engineering, vol. 8, no. 6, Article ID 066009, 2011.

[43] E. Yom-Tov and G. F. Inbar, "Detection of movement-related potentials from the electro-encephalogram for possible use in a brain-computer interface," Medical and Biological Engineering and Computing, vol. 41, no. 1, pp. 85-93, 2003.

[44] K. Fukunaga, Introduction to Statistical Pattern Recognition, Academic Press, 2013.

[45] C. M. Bishop, Neural Networks for Pattern Recognition, Oxford University Press, Oxford, UK, 1995.

[46] L. Breiman, "Arcing classifier (with discussion and a rejoinder by the author)," The Annals of Statistics, vol. 26, pp. 801-849, 1998.

[47] B. Blankertz, G. Curio, and K.-R. Muller, "Classifying single trial EEG: towards brain computer interfacing," in Proceedings of the Advances in Neural Information Processing Systems (NIPS '02), vol. 1, pp. 157-164, September 2002.

[48] F. Cincotti, A. Scipione, A. Timperi et al., "Comparison of different feature classifiers for brain computer interfaces," in Proceedings of the 1st International IEEE EMBS Conference on Neural Engineering, pp. 645-647, Capri Island, Italy, March 2003.

[49] B. Blankertz, R. Tomioka, S. Lemm, M. Kawanabe, and K.-R. Müller, "Optimizing spatial filters for robust EEG single-trial analysis," IEEE Signal Processing Magazine, vol. 25, no. 1, pp. 41$56,2008$.

[50] A. Hyvärinen and E. Oja, "Independent component analysis: algorithms and applications," Neural Networks, vol. 13, no. 4-5, pp. 411-430, 2000.

[51] S. Wang and C. J. James, "Extracting rhythmic brain activity for brain-computer interfacing through constrained independent component analysis," Computational Intelligence and Neuroscience, vol. 2007, Article ID 41468, 9 pages, 2007.

[52] S. Makeig, S. Debener, J. Onton, and A. Delorme, "Mining event-related brain dynamics," Trends in Cognitive Sciences, vol. 8, no. 5, pp. 204-210, 2004.

[53] A. T. Boye, U. Q. Kristiansen, M. Billinger, O. F. D. Nascimento, and D. Farina, "Identification of movement-related cortical potentials with optimized spatial filtering and principal component analysis," Biomedical Signal Processing and Control, vol. 3, no. 4, pp. 300-304, 2008.

[54] A. Ramos-Murguialday, D. Broetz, M. Rea et al., "Brainmachine interface in chronic stroke rehabilitation: a controlled study," Annals of Neurology, vol. 74, no. 1, pp. 100-108, 2013.

[55] A. Biasiucci, R. Leep, A. Al-Khodairy, A. Buhlmann, and J. D. R. Millán, "Motor recovery after stroke by means of BCIguided functional electrical stimulation," in Proceedings of the 5th International Brain-Computer Interface Meeting, Asilomar, Calif, USA, June 2013.

[56] J. E. Paciga, P. D. Richard, and R. N. Scott, "Error rate in five-state myoelectric control systems," Medical and Biological Engineering and Computing, vol. 18, no. 3, pp. 287-290, 1980.

[57] R. T. Lauer, P. H. Peckham, K. L. Kilgore, and W. J. Heetderks, "Applications of cortical signals to neuroprosthetic control: a critical review," IEEE Transactions on Rehabilitation Engineering, vol. 8, no. 2, pp. 205-208, 2000.

[58] M. Velliste, S. Perel, M. C. Spalding, A. S. Whitford, and A. B. Schwartz, "Cortical control of a prosthetic arm for self-feeding," Nature, vol. 453, no. 7198, pp. 1098-1101, 2008. 
[59] N. Mrachacz-Kersting, S. R. Kristensen, I. K. Niazi, and D. Farina, "Precise temporal association between cortical potentials evoked by motor imagination and afference induces cortical plasticity," The Journal of Physiology, vol. 590, no. 7, pp. 16691682, 2012.

[60] C. Haw, D. Lowne, and S. Roberts, "User specific template matching for event detection using single channel EEG," in Proceedings of the 3rd International Brain-Computer Interface Workshop and Training Course, p. 44, Graz University of Technology, Graz, Austria, September 2006.

[61] O. Bai, P. Lin, S. Vorbach, J. Li, S. Furlani, and M. Hallett, "Exploration of computational methods for classification of movement intention during human voluntary movement from single trial EEG," Clinical Neurophysiology, vol. 118, no. 12, pp. 2637-2655, 2007.

[62] C.-C. Chang and C.-J. Lin, "LIBSVM: a library for support vector machines," ACM Transactions on Intelligent Systems and Technology, vol. 2, article 27, 2011.

[63] E. Lew, R. Chavarriaga, S. Silvoni, and J. D. R. Millán, "Detection of self-paced reaching movement intention from EEG signals," Frontiers in Neuroengineering, vol. 5, 2012.

[64] P. Ahmadian, S. Sanei, L. Ascari, L. González-Villanueva, and M. A. Umilta, "Constrained blind source extraction of readiness potentials from EEG," IEEE Transactions on Neural Systems and Rehabilitation Engineering, vol. 21, no. 4, pp. 567-575, 2013.

[65] I. K. Niazi, N. Jiang, M. Jochumsen, J. F. Nielsen, K. Dremstrup, and D. Farina, "Detection of movement-related cortical potentials based on subject-independent training," Medical and Biological Engineering \& Computing, vol. 51, no. 5, pp. 507-512, 2013.

[66] N. Jiang, L. Gizzi, N. Mrachacz-Kersting, K. Dremstrup, and D. Farina, "A brain-computer interface for single-trial detection of gait initiation from movement related cortical potentials," Clinical Neurophysiology, vol. 126, no. 1, pp. 154-159, 2015.

[67] J. Ibáñez, J. I. Serrano, M. D. Del Castillo et al., "Detection of the onset of upper-limb movements based on the combined analysis of changes in the sensorimotor rhythms and slow cortical potentials," Journal of Neural Engineering, vol. 11, no. 5, Article ID 056009, 2014.

[68] S. J. Raudys and A. K. Jain, "Small sample size effects in statistical pattern recognition: recommendations for practitioners," IEEE Transactions on Pattern Analysis \& Machine Intelligence, vol. 13, no. 3, pp. 252-264, 1991.

[69] A. K. Jain and B. Chandrasekaran, "39 Dimensionality and sample size considerations in pattern recognition practice," Handbook of Statistics, vol. 2, pp. 835-855, 1982.

[70] J. Qin, Y. Li, and A. Cichocki, "ICA and committee machinebased algorithm for cursor control in a BCI system," in Advances in Neural Networks-ISNN 2005: Second International Symposium on Neural Networks, Chongqing, China, May 30-June 1, 2005, Proceedings, Part I, vol. 3496 of Lecture Notes in Computer Science, pp. 973-978, Springer, 2005.

[71] H. Lee and S. Cho, "PCA+HMM+SVM for EEG pattern classification," in Proceedings of the 7th International Symposium on Signal Processing and Its Applications (ISSPA '03), vol. 1, pp. 541-544, IEEE, Paris, France, July 2003. 


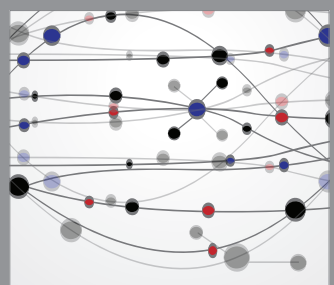

The Scientific World Journal
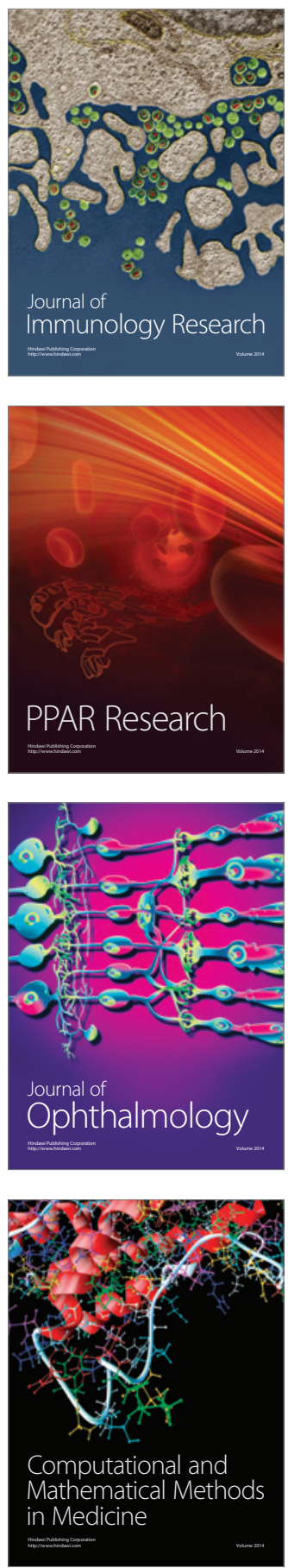

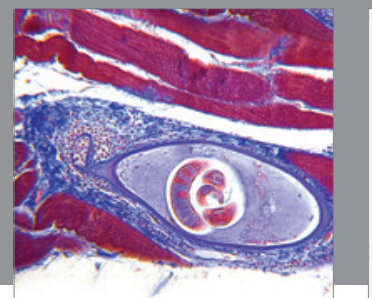

Gastroenterology

Research and Practice
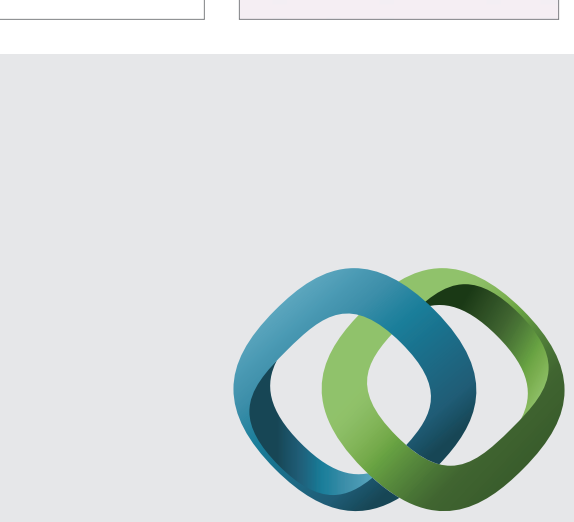

\section{Hindawi}

Submit your manuscripts at

http://www.hindawi.com
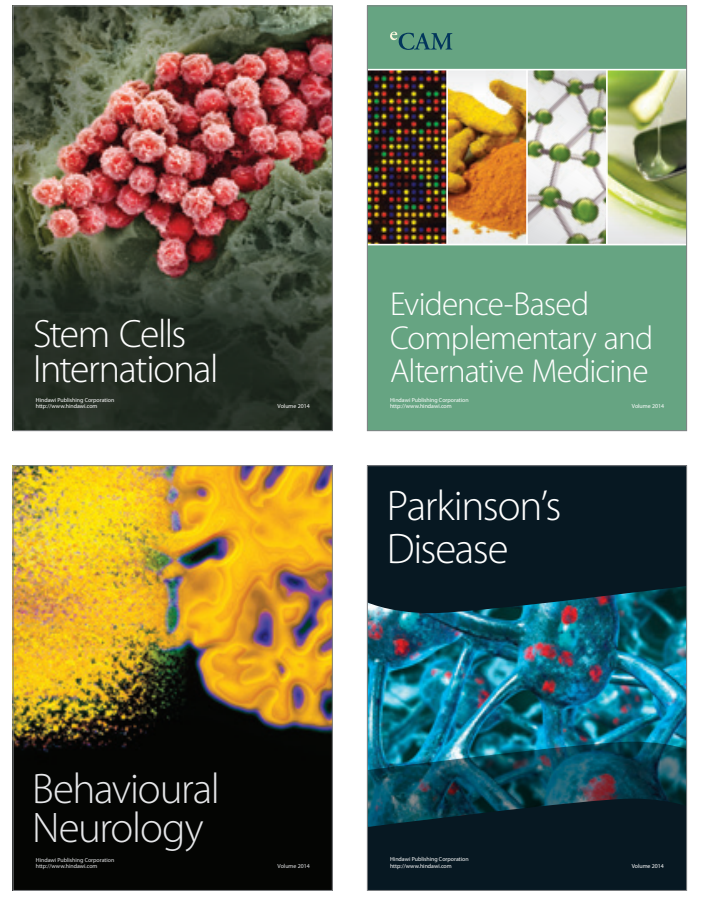
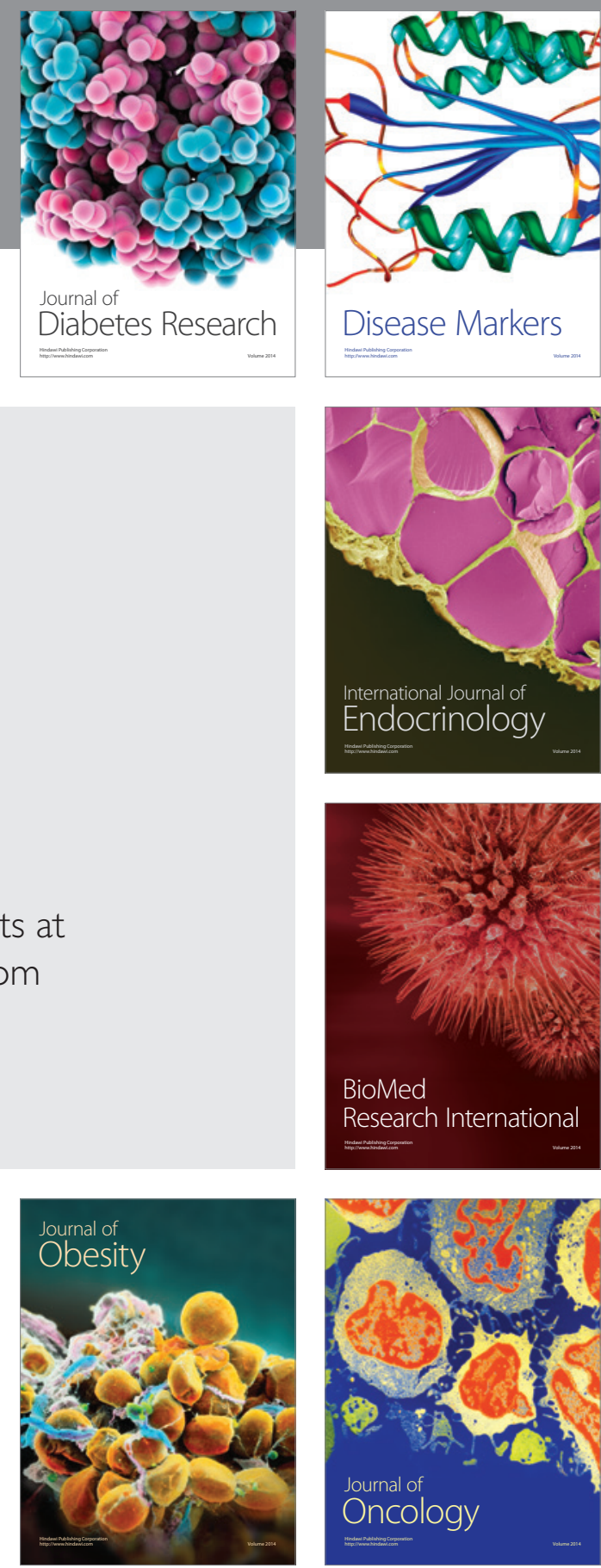

Disease Markers
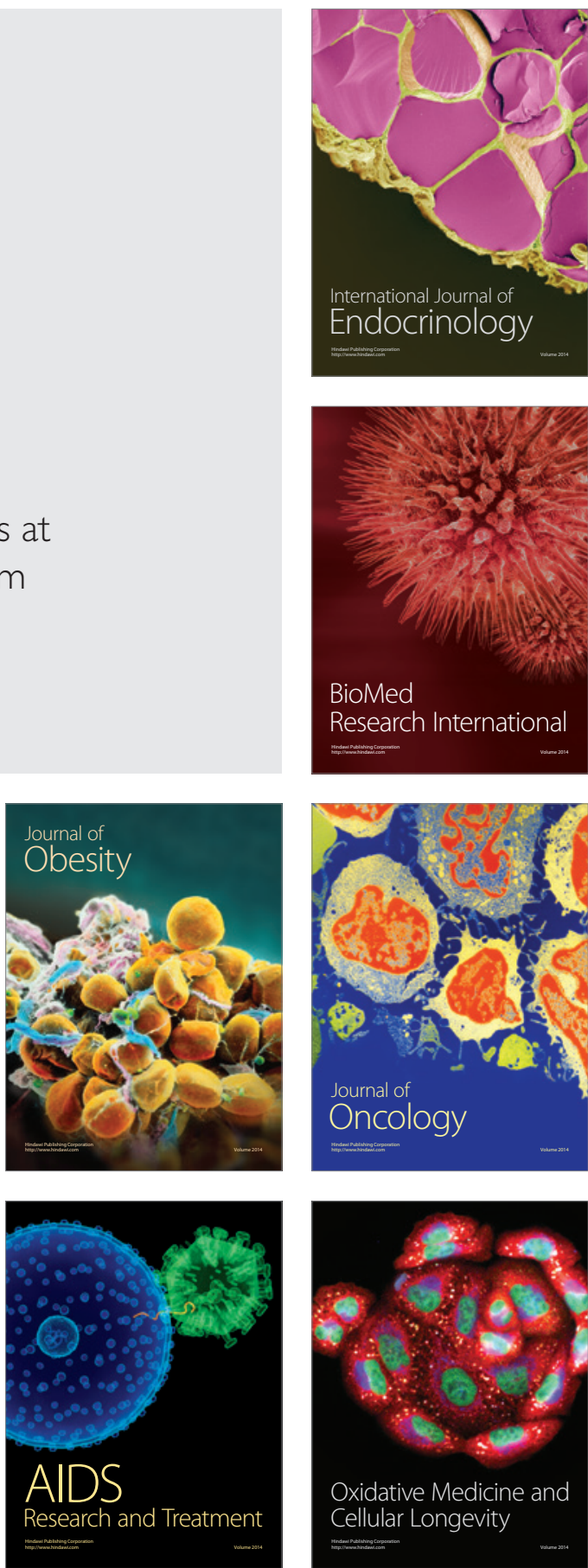